\title{
A TRANSDISCIPLINARIDADE COMO UMA RESPOSTA À SUSTENTABILIDADE
}

\section{LA TRANSDISCIPLINARIEDAD COMO RESPUESTA A LA SOSTENIBILIDAD}

\section{THE TRANSDISCIPLINARITY AS A RESPONSE TO SUSTAINABILITY}

\author{
D’AMBROSIO, Ubiratan ${ }^{1}$
}

\begin{abstract}
RESUMO: Neste trabalho examino o conceito de análises e práticas reducionistas positivocartesianas, e proponho a transdisciplinaridade como resposta necessária à sustentabilidade. $\mathrm{O}$ conhecimento dominante, socializado pelos poderosos meios de informação e comunicação não pode ignorar as várias dimensões do conhecimento: sensorial, intuitiva, emocional, mística, racional. As ciências e, consequentemente, a tecnologia atingiram seu estágio de desenvolvimento graças a análises e práticas reducionistas, fragmentadas em disciplinas, que favorecem uma percepção seletiva de problemas e, portanto, a especialização. O que se nota é que as soluções propostas não atingem a meta essencial de um enfoque integrado. Faz-se necessária uma reorientação das ciências e da tecnologia, fundada numa integração dos vários modos de conhecimento, transcendendo as culturas e as disciplinas, a partir de uma perspectiva transdisciplinar.
\end{abstract}

Palavras chave: sustentabilidade, transdisciplinaridade, ciência, tecnologia, engenharia

RESUMEN: En este trabajo se examina el concepto cartesiano reduccionista de análisis y prácticas, y proponer la transdisciplinariedad como una respuesta necesaria a la sostenibilidad. El conocimiento dominante, socializados por los poderosos medios de comunicación y la comunicación no pueden ignorar las diversas dimensiones del conocimiento: sensorial, intuitivo, emocional, racional y mística. Las ciencias y en consecuencia, la tecnología llegó a su etapa de prácticas de desarrollo a través del análisis y reduccionista, disciplinas fragmentadas, lo que favorece una percepción selectiva de los problemas y por lo tanto de especialización. Lo que cuenta es que las soluciones propuestas no cumplen con el objetivo esencial de un enfoque integrado. Es necesario que haya una reorientación de la ciencia y la tecnología, fundada en la integración de los diversos modos de conocimiento, que trasciende culturas y disciplinas, desde una perspectiva transdisciplinaria.

Palabras clave: sostenibilidad, transdisciplinariedad, ciencia, tecnología, ingeniería.

\footnotetext{
${ }^{1}$ Ubiratan D’Ambrosio é Doutor em Matemática pela Universidade de São Paulo (1963) e Professor Emérito da Universidade Estadual de Campinas/UNICAMP, Atualmente é Professor do Programa de Pós-Graduação em Educação Matemática da UNIBAN/Universidade Bandeirante de São Paulo. ubi@usp.br CV em http://lattes.cnpq.br/1531403209010948
} 


\begin{abstract}
In this paper I examine the concept of Cartesian reductionist analyzes and practices, and propose transdisciplinarity as a necessary response to sustainability. The dominant knowledge, socialized by the powerful media and communication cannot ignore the various dimensions of knowledge: sensory, intuitive, emotional, mystical, rational. The sciences and consequently the technology reached its stage of development practices through the analysis and reductionist, fragmented disciplines, which favor a selective perception of problems and therefore specialization. What we note is that the proposed solutions do not meet the essential goal of an integrated approach. There needs to be a reorientation of science and technology, founded on integration of various modes of knowledge, transcending cultures and disciplines, from a transdisciplinary perspective.
\end{abstract}

Key word: sustainability; transdisciplinarity; science, technology, engineering

\title{
INTRODUÇÃO
}

"Estando em nossa torre de marfim, o que podemos dizer? Estamos nesta torre de marfim, e nos sentimos confortáveis nela. Mas, realmente, não podemos dizer muito porque não vemos bem o mundo. Temos que sair, mas isto não é tão fácil"

Gromov 2010

Mikhail Leonidovich Gromov (nascimento 23/12/1943) é Professor do Institute des Hautes Études Scientifiques de Bûres-sur-Yvette, França e em 2009 recebeu o Prêmio Abel (equivalente a um Prêmio Nobel em Matemática) por "suas contribuições revolucionárias à geometria”. Em entrevista, Gromov reflete sobre o estado do mundo e diz:

"A Terra vai ficar sem os recursos básicos, e não podemos prever o que vai acontecer depois disso. Vamos ficar sem água, ar, solo, metais raros, para não falar do petróleo. Tudo vai, essencialmente, chegar ao fim dentro de cinquenta anos. O que vai acontecer depois disso? Estou com medo. Tudo pode ir bem se encontrarmos soluções, mas se não, então tudo pode chegar muito rapidamente ao fim!"’2

Esta é uma preocupação real, sentida por todos nós. O pessimismo de Gromov não é uma afirmação leviana, jargão próprio de catastrofistas, nem uma visão apocalíptica, de cunho religioso. Vindo de uma pessoa com seu status acadêmico, merece muita atenção.

\footnotetext{
${ }^{2}$ Entrevista de Mikhail Gromov para M. Raussen e C. Skau. Notices of the AMS, v.57, n 3, March, 2010, p.391409.
} 
A pergunta que naturalmente segue é "O que podemos fazer?" O próprio Gromov comenta, como citado na epígrafe acima.

Há muito tempo tenho utilizado uma metáfora, que chamo de "gaiolas epistemológicas", equivalente à metáfora das torres de marfim, para definir conhecimento tradicional. Apresentarei essa metáfora mais adiante neste trabalho.

\section{APELO A CIENTISTAS E ENGENHEIROS PARA LOGRAR SOCIEDADES SUSTENTÁ VEIS.}

A organização não governamental INES [International Network of Scientists and Engineers for Global Responsibility] tem feito importantes estudos sobre a responsabilidade de cientistas e engenheiros na promoção da paz e da sustentabilidade. Visite o site http://www.inesglobal.com/ines-home.phtml

No seu Newsletter de Outubro de 2004, a INES publicou uma Declaração que é um apelo aos engenheiros e cientistas para se lograr sociedades sustentáveis. A Declaração continua muito atual. ${ }^{3}$

A Declaração começa reconhecendo que o conceito de sustentabilidade é baseado em valores e no sistema de conhecimentos que deve focalizar harmonia com a natureza e não domínio sobre a natureza. Qualquer análise global do estado do mundo nos mostra que a pratica corrente de domínio sobre a natureza é falida e conduz ao colapso total do planeta.

Um processo de desenvolvimento objetivando sustentabilidade deve tomar em consideração os seguintes pontos:

- Proteção da integridade da biosfera;

- Uso eficaz de recursos;

- Auto-dependência;

- Democracia participativa;

- Comércio justo;

- Paz e não-violência.

\footnotetext{
${ }^{3}$ http://www.inesglobal.com/download.php?f=19298ee05f45d82d63a194087db8ef28
} 
Ciência e tecnologia oferecem os instrumentos que permitem atingir todos esses pontos. Mas, paradoxalmente, o que temos visto é que a ciência e a tecnologia têm tido consequiências opostas. A que se atribui isso?

As ciências e, conseqüentemente, a tecnologia atingiram seu estágio de desenvolvimento graças a análises e práticas reducionistas, fragmentadas em disciplinas, que favorecem uma percepção seletiva de problemas e, portanto, a especialização.

O que se nota é que as soluções propostas não atingem a meta essencial de um enfoque integrado. Faz-se necessária uma reorientação das ciências e da tecnologia, baseada numa integração dos vários modos de conhecimento, transcendendo as culturas e as disciplinas.

\section{SISTEMAS DE CONHECIMENTO}

Neste trabalho vou examinar o conceito de análises e práticas reducionistas e fazer uma proposta na direção apontada como necessária. O conhecimento dominante, socializado pelos poderosos meios de informação e comunicação, não pode ignorar suas várias dimensões: sensorial, intuitiva, emocional, mística, racional.

O conhecimento que se desenvolveu a partir das culturas mediterrâneas se caracteriza por ter aprofundado uma percepção do cosmos, do planeta e da natureza que vê os seres humanos como uma espécie privilegiada. A aceitação e subordinação ao Criador e a seus profetas implicam privilégios individuais e de seitas. O conhecimento é fortemente orientado para a realização dos privilégios e determina comportamentos subordinados a eles. Os sistemas de valores são relativizados e justificados pelo conhecimento.

Ao longo da história, o conhecimento originado nas culturas mediterrâneas foi, gradativamente, enaltecendo a dimensão racional e reduzindo, chegando à tentativas de eliminar, as dimensões sensorial, intuitiva, emocional e mística. Esse conhecimento impôs como a característica por excelência do ser humano a sua dimensão racional.

A teoria do conhecimento orientou-se, de acordo com essa postura, para reconhecer o racional como suporte exclusivo da ciência e da tecnologia. O conhecimento com ênfase no intuitivo é identificado como próprio das artes, o místico e o emocional com as religiões e o sensorial com o empirismo e suas conotações negativas. Repetindo, as ciências, e consequentemente a tecnologia, são caracterizadas pela dimensão racional. As demais dimensões comparecem no que são chamadas humanidades, artes e tradições, o que inclui as 
religiões e a espiritualidade no sentido amplo. As duas culturas, como discutidas por C. P. Snow, num livro clássico, são presentes.

O flanco vulnerável da racionalidade científica foi exposto de forma mais flagrante justamente pela ciência identificada como o padrão da racionalidade, que é a matemática. $\mathrm{Na}$ busca de se procurar fundamentar o conhecimento matemático e a sua geração, na transição do século XIX para o século XX, o matemático L. E. J. Brower, proposto em 1906, contrapõe-se ao logicismo de Bertrand Russell e ao formalismo de David Hilbert, rejeitando justamente o tertium non datur (lei do terceiro excluído), sobre o qual se funda grande parte do pensamento matemático. Sintetizando Brouwer e o intuicionismo simplesmente rejeitam a afirmação que "é” ou "não é” , ou em outros termos, "verdadeiro" ou "falso" sejam as únicas possibilidades. $^{4}$

Com motivação diferente, motivado pela Relatividade e pela Mecânica Quântica, o filósofo Stéphane Lupasco (1900-1988) propõe uma lógica não-aristotélica também rejeitando o tertium non datur. ${ }^{5}$ As propostas de Lupasco foram controvertidas no mudo acadêmico e retomadas e explicadas por Basarab Nicolescu, vem ganhando maior visibilidade.

Igualmente atingida foi a visão de um universo newtoniano com o surgimento das mecânicas quântica e relativística, a partir de Max Planck e Albert Einstein e com as formulações de Niels Bohr e Werner Heisenberg. ${ }^{6}$ Fundamentalmente atingido foi a percepção de uma realidade determinista e a linearidade nela implícita, obedecendo relações de causa-efeito. Abriu-se assim o caminho para as teorias geral dos sistemas e teorias do caos e da complexidade e para uma nova visão do universo.

Não menos atingida foi a visão de homem, com a percepção da essencialidade do outro no reconhecimento do seu próprio eu. Os trabalhos pioneiros de S. Freud sobre a histeria abriram o caminho para uma nova ciência da mente e do comportamento.

A partir dessas novas visões de cognição, da mente e do comportamento, e do cosmos o homem começa a se reconhecer como uma entidade individual, social, planetária e cósmica.

A civilização ocidental tem privilegiado o existencial e o factível e construído sistemas de conhecimento visando sua sobrevivência. São as ciências e a conseqüente tecnologia. Mas,

\footnotetext{
${ }^{4}$ Para uma síntese ver o verbete Foundations of Mathematics no excelente Encyclopedic Dictionary of Mathematics, by the Mathematical Society of Japan, edited by Shôkichi Iyanaga and Yukiyosi Kawada, translation reviewed by Kenneth. O. May, The MIT Press, Cambridge, 1980; p.549.

${ }^{5}$ Stéphane Lupasco. Le principe d'antagonisme et la logique de l'énergie. Prolégomènes à une science de la contradiction, Hermann \& Co., Paris, 1951..

${ }^{6}$ Para uma síntese ver o verbete quantum no Dictionary of the History of Science ed. W.F.Bynum, E.J.Browne, Roy Porter, Princeton University Press, Princeton, 1984.
} 
paradoxalmente, a sobrevivência do indivíduo, e portanto de toda a espécie, sente-se ameaçada justamente pelas intermediações criadas pela espécie.

O desenvolvimento das ciências e da tecnologia, que estão intimamente ligadas, deram origem ao que hoje se chama tecnociência, o que leva à percepção de possibilidade de extinção da espécie. A alternativa de uma espécie modificada, não mais homo sapiens sapiens, que tem sido contemplada na ficção, começa a se tornar uma possibilidade.

Pergunta-se porque esse roteiro na busca do conhecimento ocidental chegou a perspectivas tão assustadoras? Ou podemos perguntar, pura e simplesmente, porque o caminho da humanidade não tem tido sucesso? As próprias ciências reconhecem sua insuficiência para responder a essas questões básicas e para encontrar um novo caminho que garanta um futuro para a humanidade.

Vamos encontrar, metaforicamente, essa conclusão num dos mais importantes resultados científicos desse século, mais uma vez justamente na ciência que, como dissemos acima, tem sido apontada como a representante por excelência do racionalismo ocidental, que é a matemática. O destacado matemático Kurt Gödel (1906-1878) mostrou, em 1931, que é impossível provar a consistência de um sistema formal utilizando somente argumentos que podem ser formalizados no sistema. ${ }^{7}$ A busca de outros caminhos torna-se necessária. A matemática, que depende de responder verdadeiro ou falso, não encontra no seu sistema, resposta a uma questão por ela formulada nesse mesmo sistema. Ela encontra-se "engaiolada" no seu sistema e a resposta só pode ser procurada saindo da "gaiola".

O sistema dominante de conhecimento criado pela humanidade, ao longo de sua história, também não dá resposta à questão básica de sua sobrevivência.

A gaiola que hospeda a ciência moderna tem a forma de um tripé: repousa sobre: a lógica aristotélica, fundamento da matemática, que é o instrumento e a linguagem básica da ciência moderna; o determinismo newtoniano, responsável pela relação causa $\rightarrow$ efeito, fixados por leis, e, portanto, pela prioridade na procurar de uma causa para tudo o que acontece; sistemas formais, com normas e implicações rígidas e acordadas, tanto nas relações comunicativas quanto nas relações sociais, suporte das legislações. O científico e o social são faces de uma mesma moeda.

Há propostas de novos pilares. Basarab Nicolescu propõe o tripé dos níveis de realidade, da lógica do terceiro incluído e da complexidade, como metodologia da pesquisa

\footnotetext{
${ }^{7}$ Kurt Gödel: "Über formal unentscheidbase Sätze der Principia Mathematica und verwandter Susteme I", Monatshefte für Mathematik und Physik, vol.38 (1931), pp.173-198. O trabalho é muito técnico, difícil de ser entendido por não especialistas. Uma versão mais accessível encontra-se em Ernest Nagel and James R. Newman: Gödel's Proof, New York University Press, New York, 1958 , p.550.
} 
transdisciplinar. ${ }^{8}$ Os níveis de realidade são uma expressão da mecânica quântica e da relatividade, que foram responsáveis por mostrar a insuficiência de um dos tripés da ciência moderna, o determinismo newtoniano. A lógica do terceiro incluído surge, com Brouwer e depois com Lupasco, do reconhecimento da complexidade de fatores que intervém no curso da realidade. Hoje, a teoria das possibilidades desafia o próprio terceiro incluído. A complexidade é o reconhecimento de uma multiplicidade de fatores desconhecidos, imprevisíveis e mesmo sendo auto-criados e auto-exterminados no instante, como mostram os avanços da virologia. Substituir três pilares sobre o qual repousa a ciência moderna pelos pilares que mostraram a sua insuficiência resulta em facilitar a credibilidade nos meios acadêmicos, mas pode resultar na criação de uma outra gaiola. È o drama das revoluções religiosas, científicas, conceituais, sociais e políticas, como a história nos mostra tão bem.

\section{A METÁFORA DAS GAIOLAS EPISTEMOLÓGICAS}

As disciplinas são como conhecimento "engaiolado" na sua fundamentação, nos seus critérios de verdade e de rigor, nos seus métodos específicos para lidar com questões bem definidas e com um código linguístico próprio, inacessível aos não iniciados.

Os detentores desse conhecimento são como pássaros vivendo em uma gaiola: alimentam-se do que lá encontram, voam só no espaço da gaiola, comunicam-se numa linguagem só conhecida por eles, procriam e repetem-se, só vendo e sentindo o que as grades permitem, como é comum no mundo acadêmico. O que é mais grave, são mantidos pelos que possuem as gaiolas para seu entretenimento, como é o caso das artes, ou para seu benefício, como é o caso das ciências e da tecnologia. Obviamente, a crítica interna é limitada e exclui o questionamento da própria existência da gaiola. A crise provocada por Kurt Gödel é ilustrativa dessa metáfora. Em 1931, ele mostrou que não há qualquer método de prova formal que possa demonstrar todas as verdades da matemática, nem mesmo a teoria dos números naturais. $^{9}$

O trabalho de Gödel causou enorme desconforto e incertezas entre os matemáticos que tinham sensibilidade para as questões de fundamentos. Certamente pela sua dificuldade, e para conveniência dos guardiões das gaiolas, o trabalho foi ignorado pela maioria dos matemáticos, que continuam tranquilos na sua gaiola, beneficiando-se do suporte dos guardiões da gaiola e baseando seu fazer matemático nos critérios de rigor vigentes. $\mathrm{O}$

\footnotetext{
${ }^{8}$ Basarab Nicolescu, O Manifesto da Transdisciplinaridade. Triom : São Paulo, 1999.

9 Op. cit. em Nota 6.
} 
conceito de verdade matemática e a sua inegável beleza serviram e continuam servindo de argamassa para fazer subir o edifício da pesquisa matemática ... mesmo que o alicerce tenha sido fragilizado. Essas observações também se aplicam às demais áreas do conhecimento. Num trabalho anterior discuto outros exemplos, inclusive sobre artes ${ }^{10}$.

A capacidade de observação e interpretação é subordinada à práticas e metodologias bem definidas. Mas nem mesmo podem saber de que cor a gaiola é pintada por fora. Isso equivale ao que Gromov diz "não podemos dizer muito porque não vemos bem o mundo", citado acima.

A organização do conhecimento em disciplinas tem suas origens na antiguidade. ${ }^{11}$ Vai tomando os aspectos das disciplinas típicas de um departamento tradicional das universidades a partir do século XVI e só vai se articular com as características atuais no século XIX, particularmente quando se organiza a Universidade de Berlim. A necessidade de multidisciplinas já havia sido apontada por Fontenelle no século XVII e as interdisciplinas começam a ser identificadas no século XIX. Mas nada altera a metáfora. Pode-se identificar as multidisciplinas como uma justaposição de gaiolas disciplinares (como é praticada nas chamadas grades curriculares da educação) e as interdisciplinas são, metaforicamente, a passagem de uma gaiola para outra eventualmente criando um "viveiro", na verdade uma gaiola maior.

O sistema de conhecimentos dominante, denominado Ciência Moderna e que se desenvolveu a partir das culturas mediterrâneas, caracteriza-se por ter aprofundado uma percepção do cosmos, do planeta e da natureza que vê os seres humanos como uma espécie privilegiada, detentores de razão. Esse conhecimento acarreta um comportamento ditado por privilégios. Os vários corpos de conhecimento, estruturados segundo a dimensão racional, passaram a ser denominados ciências, que acabou sendo identificada com conhecimento. As demais dimensões comparecem no que são chamadas as tradições.

A civilização ocidental tem privilegiado o existencial e o factível e construído sistemas de conhecimento visando sua sobrevivência.

\footnotetext{
${ }^{10}$ Ubiratan D'Ambrosio Teoria da Relatividade, o Princípio da Incerteza, O Expressionismo, org. J.Guinsburg, Editora Perpectiva, São Paulo, 2002; pp.103-120.

${ }^{11}$ Ver o livro recente de G. E. R. Lloyd, Disciplines in the Making. Cross-cultural Perspectives on Elites, Learning and Innovation, Oxford: Oxford University Press, 2009.
} 


\section{DAS DISCIPLINAS À TRANSDISCIPLINARIDADE}

É na história do conhecimento que vamos identificar as distorções e os novos caminhos possíveis. Obviamente, essa história não pode se restringir a uma visão parcial, epistemologicamente comprometida. Torna-se assim necessário o diálogo que começa a se abrir entre as ciências e as tradições.

A primeira lição que aprendemos da história é que a busca de sobreviver se complementa com a busca de transcender o existencial (passado e futuro) e o factível (explicável e inexplicável). A busca de compreensão do mundo na sua integralidade exige que as dimensões de sobrevivência e de transcendência se complementem.

A organização atual dos diversos corpus de conhecimento repousa nas disciplinas, caracterizadas pelo desenvolvimento de métodos específicos para conhecer objetos de estudo bem definidos.

Rapidamente, o conhecimento disciplinar mostrou-se insuficiente para lidar com os complexos fenômenos da realidade e pratica-se um modelo multidisciplinar, no qual se procura reunir resultados obtidos mediante o enfoque disciplinar para lidar com situações mais complexas. Igualmente, o enfoque multidisciplinar foi incapaz de explicar e de lidar com o crescente reconhecimento da complexidade dos fenômenos naturais e sociais.

É curioso notar que a partir de uma visão global dos fenômenos foi proposta a organização disciplinar dos conhecimentos e imediatamente a multidisciplinar. Esse próprio enfoque criou a possibilidade de se reconhecer a complexidade dos fenômenos, que resistiam aos métodos das várias disciplinas isoladamente.

Surge então a interdisciplinaridade, na qual não apenas se transferem e se combinam resultados de algumas disciplinas, mas também se combinam métodos de várias disciplinas e, conseqüentemente, se identificam novos objetos de estudo.

Chega-se assim a possibilidades de explicações de inúmeros fenômenos e de realizações notáveis. Os espantosos avanços das ciências e da tecnologia, produto da interdisciplinaridade, dão ao homem a ilusão de omnipotência e de omnisciência.

Há limites para o conhecimento? Haverá a possibilidade de um sistema total de conhecimento ou, como costumam dizer os físicos, de uma teoria de tudo?

Mas mesmo na prática interdisciplinar, que hoje, com raras exceções, está presente na pesquisa científica, e que procura explicações finais, não se supera a angústia da ameaça de extinção que pesa sobre a humanidade. 
Haverá a possibilidade de outras opções além da interdisciplinaridade? Não se pode excluir a possibilidade de existirem outros sistemas de conhecimento nos quais a fragmentação do saber não tenha sido tão rígida quanto o que vemos no sistema de conhecimento ocidental. Esses sistemas de conhecimento, supérstites em algumas sociedades, muitos aprimorados, outros desfigurados pela exposição com a civilização ocidental, encontram-se nas tradições. É bem possível que esses sistemas poderão contribuir para a superação do impasse existencial.

Do encontro das ciências e das tradições resultam transformações profundas dos sistemas de explicações. Essas transformações têm sido notadas no desenvolvimento científico e tecnológico, nos sistemas de comunicação e de transporte, nos meios de produção e no próprio conceito de propriedade. Um questionamento freqüente se refere à universalidade dessas transformações. Há indicadores de que esse encontro é efetivamente um movimento planetário, prenúncio de uma civilização planetária.

O conhecimento é então encarado como modos, estilos, técnicas de explicar, de conhecer, de lidar com a realidade como ela se manifesta em distintos ambientes naturais e culturais. Obviamente, esses modos, estilos e técnicas não se realizam no modelo disciplinar, nem mesmo nos seus variantes da multidisciplinaridade e da interdisciplinaridade. Exigem uma visão transdisciplinar do conhecimento.

A transdisciplinaridade leva o indivíduo a tomar consciência da essencialidade do outro e da sua inserção na realidade social, natural e planetária, e cósmica. Uma conseqüência imediata da essencialidade é que a inserção só pode se dar através de um relacionamento de respeito, solidariedade e cooperação com o outro, conseqüentemente com a sociedade, com a natureza e com o planeta, todos e tudo integrados na realidade cósmica. Esse é o despertar da consciência na aquisição do conhecimento. A grande transformação pela qual passa a humanidade é o encontro do conhecimento e da consciência. A transdisciplinaridade procura entender e propor como o ser humano, um fato biológico, material, atinge a sobrevivência e a transcendência, características da qualidade de ser humano, um fato espiritual. ${ }^{12}$

\section{A PROPOSTA DA TRANSDISCIPLINARIDADE}

Qual a oportunidade que a academia tem tido de refletir sobre essas questões? É possível que o "Fórum de Ciências e Cultura da UNESCO", que se reuniu em Veneza em 
1986, tenha sido a primeira grande oportunidade de se propor uma visão transdisciplinar. O tema do fórum, "Encontro das Ciências e das Tradições", e a composição de sua participação foram, inegavelmente, fatores essenciais para uma nova postura com relação ao conhecimento. A Declaração de Veneza, que foi um resultado do Fórum, abriu as possibilidades de reflexões transdisciplinares.

Uma importante consequiência do Fórum foi a criação, em 1987, pelo físico Basarab Nicolescu, do CIRET/Centre international de recherches et études transdisciplinaires, que, sob sua direção tem sido um catalizador de pesquisas e ações transdisciplinares.

Claro que a transdisciplinaridade não constitui uma nova filosofia, nem uma ciência das ciências e, muito menos, uma nova metafísica nem uma nova postura religiosa. Nem é, como muitos insistem em caracterizá la, um modismo. O essencial na transdisciplinaridade reside numa postura de reconhecimento que não há espaço e tempo culturais privilegiados que permitam julgar e hierarquizar, como mais correto ou mais certo ou mais verdadeiro, complexos de explicação e convivência com a realidade que nos cerca. Ao reconhecer que não se pode atingir um conhecimento final e, portanto, dever estar em permanente evolução, a transdisciplinaridade repousa sobre uma atitude aberta, de respeito mútuo e, mesmo, humildade, com relação a mitos, religiões e sistemas de explicações e conhecimentos, rejeitando qualquer tipo de arrogância e prepotência.

A transdisciplinaridade é, na sua essência, transcultural. Exige a participação de todos, vindo de todas as regiões do planeta, de tradições culturais e formação e experiência profissional as mais diversas. Esse aspecto da transdisciplinaridade foi destacado nos dois fóruns de "Ciência e Cultura", realizados em Vancouver, Canadá, em 1989, e em Belém, do Pará, em 1992. O Fórum de Veneza focalizou o conhecimento moderno na própria sede de sua origem, As dependências em que nos reunimos haviam sido freqüentadas por Galileo.

Uma das recomendações da Declaração de Veneza é que novos encontros fossem realizados em diferentes ambientes e com enfoques diversos. Particularmente importante seria examinar o enorme fosso criado por um alto padrão de desenvolvimento à custa da supressão de estilos tradicionais de vida, e a questão de sustentabilidade.

O Fórum em Vancouver privilegiou o exame da situação de uma das regiões mais desenvolvidas do mundo, no conceito moderno - uma exorbitância de construções --

${ }^{12}$ Ubiratan D'Ambrosio; Transdisciplinaridade, Editora Palas Athena, São Paulo, 2009. 
contrastando com a eliminação de uma das mais fortes tradições nativas, as populações indígenas do Pacífico. O Fórum de Belém, realizado no encontro dos hemisférios Norte e Sul, onde uma realidade de desenvolvimento acentuado convive com as práticas mais tradicionais e com as populações indígenas.

As Declarações de Vancouver e de Belém apontam para uma visão ampla da transdisciplinaridade, focalizando a sustentabilidade econômica, cultural e natural. ${ }^{13}$

A essência da proposta transdisciplinar parte de um reconhecimento que a atual proliferação das disciplinas e especialidades acadêmicas e não-acadêmicas conduz a um crescimento incontestável do poder associado a detentores desses conhecimentos fragmentados, podendo assim agravar a crescente iniqüidade entre indivíduos, comunidades, nações e países. Além disso, o conhecimento fragmentado dificilmente poderá dar a seus detentores a capacidade de reconhecer e enfrentar os problemas e situações novas que emergem de um mundo a cuja complexidade natural acrescenta-se a complexidade resultante desse próprio conhecimento transformado em ação que incorpora novos fatos à realidade, através da tecnologia.

Eliminar arrogância, inveja, prepotência e adotar respeito, solidariedade, cooperação é a idéia de base na busca do conhecimento transdisciplinar. Busca-se um pacto moral entre todos os homens definitivamente interessados numa nova perspectiva de futuro para a humanidade, através de uma ética maior.

\section{O princípio essencial da transdisciplinaridade:}

reconhecer as dimensões sensorial + mística + emocional + intuitiva + racional do conhecimento e a integridade mente + corpo + cosmos, dando suporte a um comportamento subordinado à ética maior de respeito, solidariedade $e$ cooperação.

\section{REFERÊNCIAS BIBLIOGRÁFICAS}

D'AMBROSIO, Ubiratan (Org.). Declarações dos Foruns de Ciência e Cultura da UNESCO (Veneza, Vancouver e Belém e a Carta da Transdisciplinaridade), Textos Universitários. Brasília: Ed. da Universidade de Brasília, 1994. , Ubiratan. Transdisciplinaridade. São Paulo: Ed. Palas Athena, 2009.

\footnotetext{
${ }^{13}$ Ubiratan D'Ambrosio (organizador): Declarações dos Foruns de Ciência e Cultura da UNESCO (Veneza, Vancouver e Belém e a Carta da Transdisciplinaridade), Textos Universitários, Editora da Universidade de Brasília, 1994
} 
Ubiratan. Teoria da Relatividade, o Princípio da Incerteza, O Expressionismo, org.

J.Guinsburg, Ed. Perpectiva, São Paulo, 2002; p.103-120.

DICTIONARY OF THE HISTORY OF SCIENCE. Ed. W.F.Bynum, E.J.Browne, Roy

Porter. Princeton: Princeton University Press, 1984.

FOUNDATIONS OF MATHEMATICS. Encyclopedic Dictionary of Mathematics. By the Mathematical Society of Japan, edited by Shôkichi Iyanaga and Yukiyosi Kawada, translation reviewed by Kenneth. O. May, The MIT Press, Cambridge, 1980; p.549.

GÖDEL, Kurt. "Über formal unentscheidbase Sätze der Principia Mathematica und verwandter Susteme I". Monatshefte für Mathematik und Physik, vol.38 (1931), p.173198.

GROMOV, Mikhail. Entrevista. Por M. Raussen e C. Skau. Notices of the AMS, v. 57, n 3, March 2010, p.391-409.

INES [International Network of Scientists and Engineers for Global Responsibility].

Declaração. Disponível em:

<http://www.inesglobal.com/download.php?f=19298ee05f45d82d63a194087db8ef28>

Acessado em: fevereiro de 2011.

LLOYD, G. E. R.. Disciplines in the Making. Cross-cultural Perspectives on Elites, Learning and Innovation. Oxford: Oxford University Press, 2009.

LUPASCO, Stéphane. Le principe d'antagonisme et la logique de l'énergie. Prolégomènes à une science de la contradiction. Hermann \& Co., Paris, 1951.

NEWMAN, James R. \& NAGEL, Ernest. Gödel's Proof. New York: New York University Press, 1958, p.550.

NICOLESCU, Basarab. O Manifesto da Transdisciplinaridade. Triom : São Paulo, 1999. 